$$
\begin{array}{cccc}
S \text { sciendo } & \text { International Conference KNOWLEDGE-BASED ORGANIZATION } \\
\text { Vol. XXV } & \text { No } 1 & 2019
\end{array}
$$

\title{
STUDY REGARDING THE EMOTIONAL SELF - AWARENESS AND EMOTIONAL SELF - CONTROL ON MANAGERS ACTIVITY
}

\author{
Hortensia GORSKI*, Diana Elena RANF** \\ *Romanian-German University, Sibiu, Romania \\ **“Nicolae Bălcescu" Land Forces Academy, Sibiu, Romania \\ tenzig11@yahoo.com, ranfd@yahoo.com
}

\begin{abstract}
This article arose from the desire to connect the concept of emotional intelligence with the business environment to identify the level of knowledge in the field and the effects it produces in this area. Because the concept is very complex, which includes many components focused on several approaches, such as: awareness of emotions, awareness of other people's emotions, understanding emotions and the ability to manage both their own emotions and others, the article focuses on just two components. Thus, the research is aimed at identifying among managers the importance that they attach to the awareness of emotions, as well as how they manage their emotions. Success in professional life depends not only on technical, economic knowledge, but to a great extent on the management of tense situations, and how it reacts in these cases.
\end{abstract}

Keywords: managers, emotions, self-awareness, self-control, emotional intelligence

\section{Introduction}

Emotions cannot be avoided, they are part of our lives and have physical, mental, behavioural, and social effects, but what is important is how we relate to them. We have the power to amplify or diminish our emotions. Avoiding, ignoring, suppressing, hiding, repressing emotions are not proper approaches. Emotional awareness, emotional self-control, a correct and objective evaluation of the situations we go through - both in personal and professional life - are very important ingredients for success. Often, our perception of a situation is not correct and, as a result, being overwhelmed by the multitude and magnitude of emotions, our reaction is not a constructive one. If we could look at situations from a different perspective, change the way we relate to them, it would be possible to identify viable ways to transform problems, threats, stressful situations into opportunities.
The ability to recognize and manage emotions and remain effective even in stressful situations influences not only the quality of our personal life, but also brings success in our professional life.

The incapacity to control hostility and explosive, choleric personality generates resentment and destroys long-term relationships with those around us subordinates, colleagues, bosses, and so on. It is impossible for a manager to lead others if he/she is not able to manage successfully his/her own emotions. Emotional selfawareness and emotional self-control have favourable effects not only on managers' decisions, but also on interpersonal relationships.

Emotional self-control has direct and propagated influences on how we relate to failures, mistakes, and how we manage to turn them into beneficial experiences from which to learn. People with a high level of emotional self-control take responsibility 
for their failures. They take responsibility and do not blame the external environment or other people (employees, colleagues), do not seek for someone or something to blame, but correctly assess the root causes, with the clear desire to identify viable solutions.

\section{Emotional self-awareness and} emotional self-control

Research related to emotional intelligence began well before being specifically recognized under this name and was the foundation for the construction of the current concept. Edward Thorndike, Howard Gardner, Wayne Leon Payne, Peter Salovey and John Mayer, Reuven Bar-On, David Goleman, Travis Bradberry and Jean Greaves - are just some of the personalities who have made an important contribution to developing the concept of emotional intelligence. We have grounded the concept of emotional intelligence in our work "The role of emotional intelligence in supporting flexibility, in the context of an organizational culture that facilitates change" published in the book "Flexibility and robustness in creating and internalizing specific managerial culture values in organizations in the field of defence and security" [5]. However, we are not dealing with these issues in the present paper.

Emotional intelligence affects our personal life and the workplace [6].

Correlational and regression analyses conducted by two Australian researches revealed that "EI, specifically the capacity to perceive emotions, was able to predict effective leadership." However, the authors suggest caution "in making too wide a generalization of these findings, as the sample size is small'"[4].

Numerous conceptual definitions of EI have been formulated over time [5]. If we analyse them, we find that, in fact, they all include an awareness of one's own emotions; an awareness of emotions in others; an understanding of emotions; and the ability to manage one's own emotions and the emotions of others. The four components or the Four-Branch model of EI are: the capacities to (a) perceive emotions, (b) use emotions to facilitate thought, (c) understand emotions, and (d) manage emotions [2].

In the article Emotional Intelligence: Toward Clarification of a Concept, Cary Cherniss highlights some very important premises: (1) Emotions play an important role in life; (2) People vary in their ability to perceive, understand, use, and manage emotions; (3) These differences affect individual adaptation in a variety of contexts, including the workplace [2].

Self-awareness is considered the starting point for emotional intelligence discovery [10], [1], [3]. Self-Awareness identify "involves the process of identifying your experience as either a thought or a feeling" [3].

First, self-awareness is about being honest with yourself. Self-awareness include elements like emotional awareness, selfassessment, self-confidence and is the first step in mastering our personal and professional life. We are living and working in a world of change. It is difficult to make changes and almost impossible to be a change catalyst without being aware of our emotions, feelings, sensations, thoughts, words, reactions, habits, beliefs, behaviour.

Emotional Self-Awareness is ,the basic foundation on which emotionally intelligent behaviour germinates and refers to the ability to read one's own emotions and recognizing their impact to guide decisions." [11]. According to Daniel Goleman, Emotional Self-Awareness „is the ability to understand your own emotions and their effects on your performance. "Understanding emotions increases the likelihood to manage them. Emotional SelfControl ,is the ability to keep disruptive emotions and impulses in check, and to maintain your effectiveness under stressful or even hostile conditions"[9]. A central process in the emergence of self-control of emotions is the regulation of attention [7]. 


\section{Research Methodology}

This paper is part of a more comprehensive study regarding emotional intelligence.

\subsection{Sample and Data Collection}

The purpose of this paper is to describe and analyse the perception of managers on emotional awareness, self-awareness, component of EI, and emotional selfcontrol, competence of the selfmanagement component.

A questionnaire-based research was conducted in April-May 2018, considering the population of private companies micro-enterprises, SMEs and large companies) from Sibiu County.

For this study managers aged 26-65 were surveyed.

A total of 98 questionnaires out of 120 have returned, all of them filled in by managers from Sibiu County.

Most of the points were measured on a five point Likert-type scale, where $1=$ Not important and 5=Very important.

Prior to the administration of the questionnaire, an interview was conducted with 5 managers, in which the same questions were used to test it.

IBM SPSS Statistics 19 for Windows and Microsoft Excel 2010 was used to process, analyse, and present the data.

\subsection{Results}

The questions in the questionnaire respond to the purpose of research and aim at objectives such as: identifying the impact of emotions on personal and professional life (emotion awareness), identifying the importance given by managers to emotion management (control of emotions), and analysing how to handle tense situations and managers' reaction to these.

- The impact of emotions on personal and professional life

Concerning the awareness of the impact of emotions on personal life (Figure 1.a), we can see that $93 \%$ of the respondents are very aware and greatly aware of the impact that emotions can have on their personal lives. Only $7 \%$ of them feel that they do not occupy an important place.
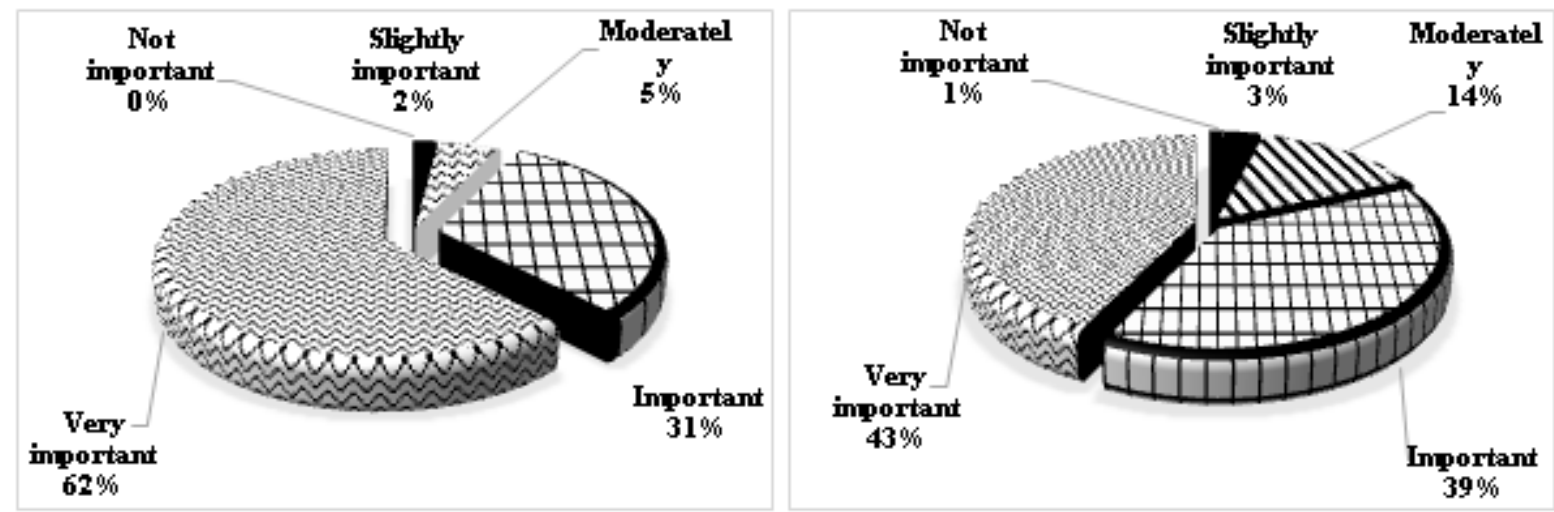

Figure 1. The impact of emotions on personal and professional life

On the other hand, if we are talking about the awareness of the impact of emotions on professional life (Figure 1.b) we notice that $82 \%$ of respondents are aware and very aware of the impact that emotions can have on their professional lives. Only 4\% say they are not important. If we consider the obtained scores, we see that, according to the respondents, the emotions have a greater influence on the personal life than on the professional life $(4,5$ and 4,2 respectively).

- The importance of managing positive and negative emotions for professional success 


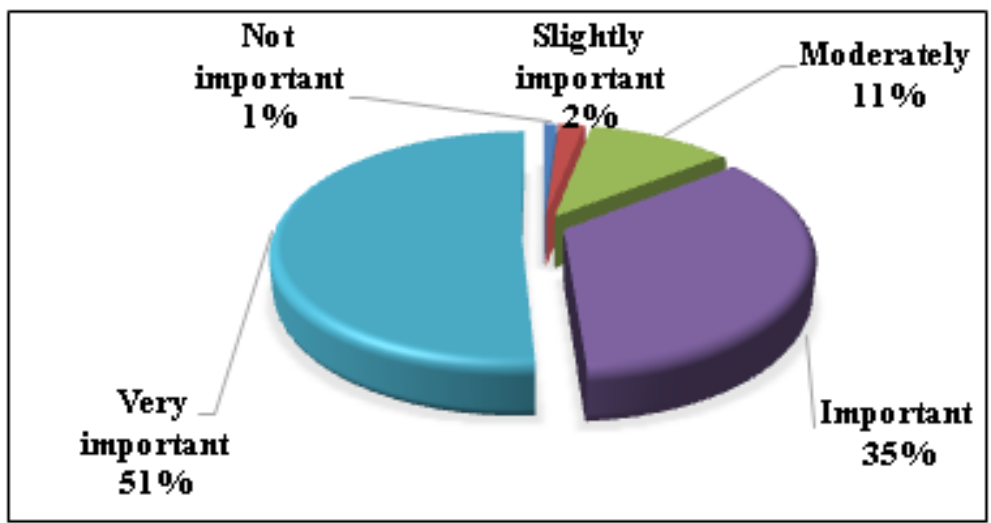

Figure 2. The importance given by managers to managing emotions

As we can see in figure 2 , we note that $86 \%$ of respondents think it is very important and important to manage their emotions in order to have success in their career. After emotion awareness, they also consider it important to manage the emotions. The obtained score is 4.32 .

- $\quad$ The way to manage tense situations

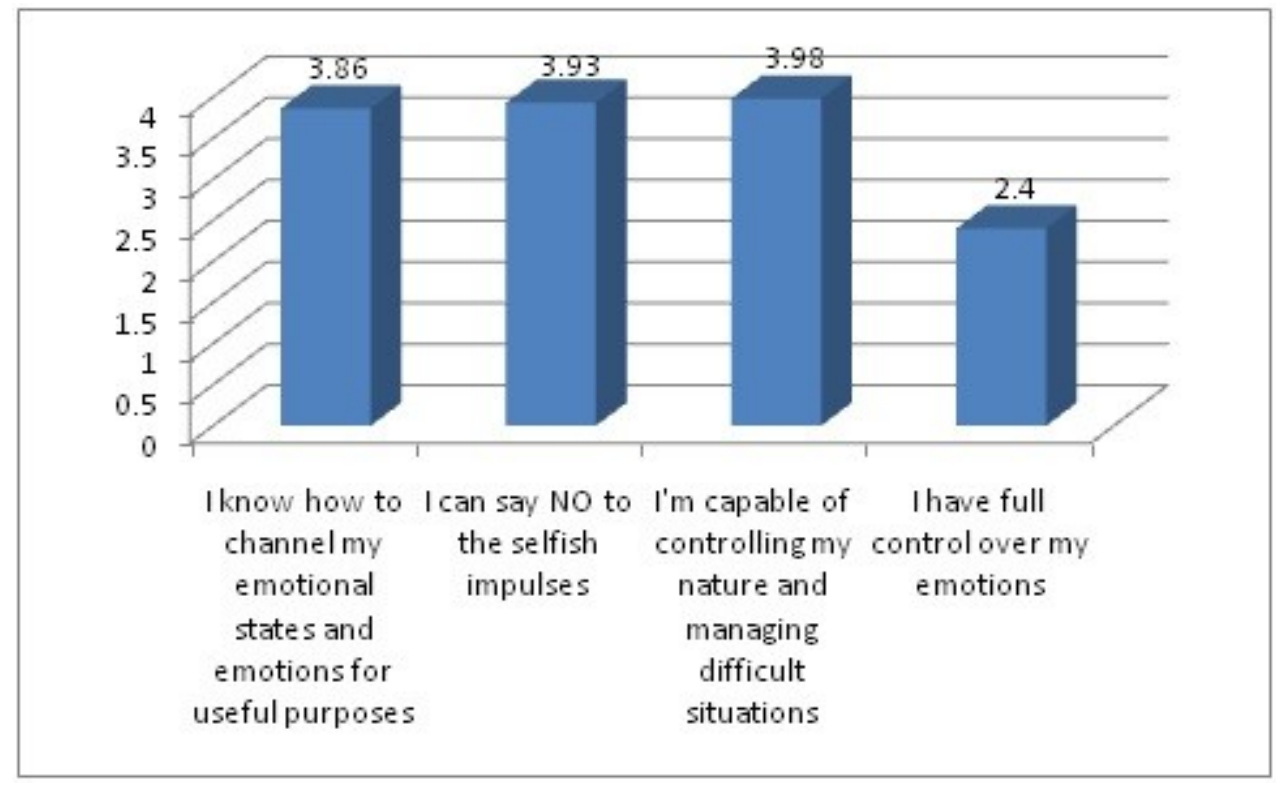

Figure 3. Managing tense situations by managers

After identifying the importance of emotion awareness and their control, we also wanted to identify managers' reaction to tense situations. Regarding the ways of managing emotions (Figure 3), respondents are generally able to control their nature and manage difficult situations (3.98), but also to deny egoistic impulses (3.93) to a great extent. However, they have admitted that they have only a small degree of total control over their emotions (2.40). In order to identify possible causes, we have continued to test their reaction to tense moments in their professional activity. 


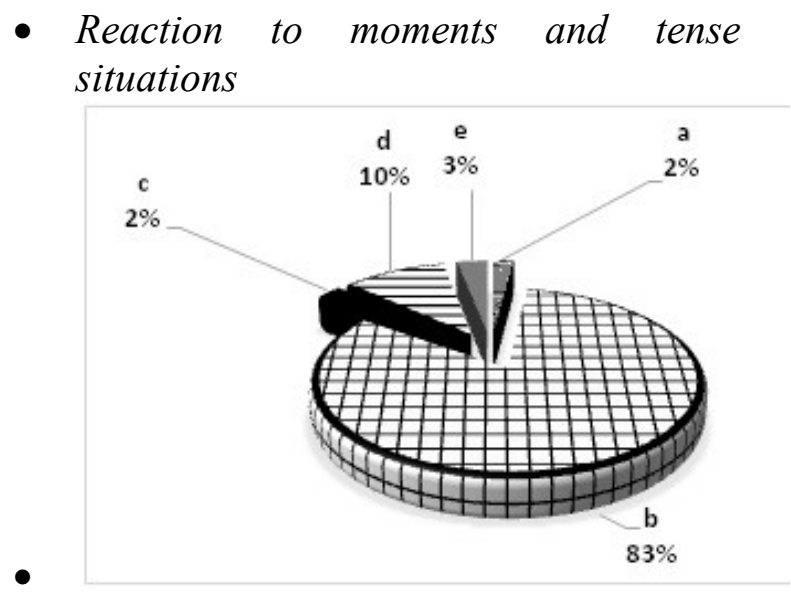

a - I cannot control my emotions - I'm angry, agitated, I get angry, and that's what I see b - I make great efforts to replace feelings of anger, irritation with calmness, relaxation $\mathrm{c}$ - Moderately

d - I succeed without too much effort to replace feelings of anger, irritation with calmness, relaxation

e - I am relaxed and act calmly

Figure 4. Reaction of managers to tense moments

Figure 4 shows that $83 \%$ of respondents are making great efforts to replace the feelings of anger and relaxation, and only $3 \%$ of them are relaxed and act calmly in tense moments. The $10 \%$ percentage of managers who manage without effort to replace the feelings of anger, mania, and calm relaxation show that managers do not have full control over emotions, as shown in figure 3 .

\section{Conclusions}

First of all, it is important to emphasize that the findings of this research cannot be taken as definite evidence because there are several limitations: a regional area; the sample size; sampling based availability (the subjects from the sample do not correspond to the statistical reality in terms of representation by type of company small, medium and large).

Despite these limitations, in our opinion, this research provides important information both from theoretical and practical perspectives. The theoretical and empirical results of this study could make an important contribution to related literature.

One of the most important contributions relates to the two competences of emotional intelligence, self-awareness and selfmanagement and how they affect the activity of the managers in the county of Sibiu.

Although managers consider emotion awareness and control of emotions as being important for the professional life, they admit they do not have full control over emotions, and that they hardly manage to react calmly in tense situations.

This conclusion demonstrates that managers still do not have the tools to control emotions by opening new research directions such as: identifying the link between emotion management and organization outcomes, identifying the main methods/tools/ways to shape/educate managers in terms of emotion control.

\section{References}

[1] Bar-On, R., The Bar-On Model of Emotional - Social Intelligence, Psicothema 18 Suppl: 13 - 25 February 2006: https://www.researchgate.net/ publication/6509274_The_Bar-On_Model_of_Emotional-Social_Intelligence.

[2] Cary Cherniss, Emotional Intelligence: Toward Clarification of a Concept, Industrial and Organizational Psychology 3(2):110 - 126, DOI: 10.1111/j.17549434.2010.01231.x, June 2010. 
[3] Darwin B. Nelson, Gary R. Low, Emotional Intelligence: Achieving Academic and Career Excellence in College and in Life, 2nd Edition, Pearson, 2011.

[4] David Rosete, Joseph Ciarrochi, Emotional intelligence and its relationship to workplace performance outcomes of leadership effectiveness, Leadership \& Organization Development Journal, Vol. 26 Issue: 5, 2005, pp.388-399.

[5] Gorski Hortensia, Ranf Diana, The role of emotional intelligence in supporting flexibility, in the context of an organizational culture that facilitates change, title of the book: Flexibility and robustness in creating and internalizing specific managerial culture values in organizations in the field of defence and security, Nicolae Balcescu Land Forces Academy Publishing Houuse, Sibiu, 2019, pp 50-69.

[6] Jeremy Bolton, A Step by Step Guide on How to Master Your Emotions, Raise Your Self Awareness, and Improve Your EQ, CreateSpace Independent Publishing Platform 2017.

[7] Kopp, C. B., Commentary: The codevelopments of attention and emotion regulation. Infancy, 3(2), 2002, 199-208.

[8] Mayer, J. D., Roberts, R. D., \& Barsade, Human abilities: Emotional intelligence. Annual Review of Psychology, 59, 2008, 507-536.

[9] Richard Boyatzis, Richard J Davidson, Vanessa Druskat, George Kohlrieser Daniel Goleman, Emotional Self-Control: A Primer, More Than Sound LLC Publisher, 2017.

[10] Salovey, Peter, and John D. Mayer "Emotional Intelligence. Imagination, Cognition, and Personality :" 185-211, 1990, DOI: 10.2190/DUGG-P24E-52WK-6CDGE-mail Citation ».

[11] Singh Kavita, Developing human capital by linking emotional intelligence with personal competencies in Indian business organizations, Journal of Business Science and Applied Management, Volume5, Issue 2, 2010, pp. 34. 